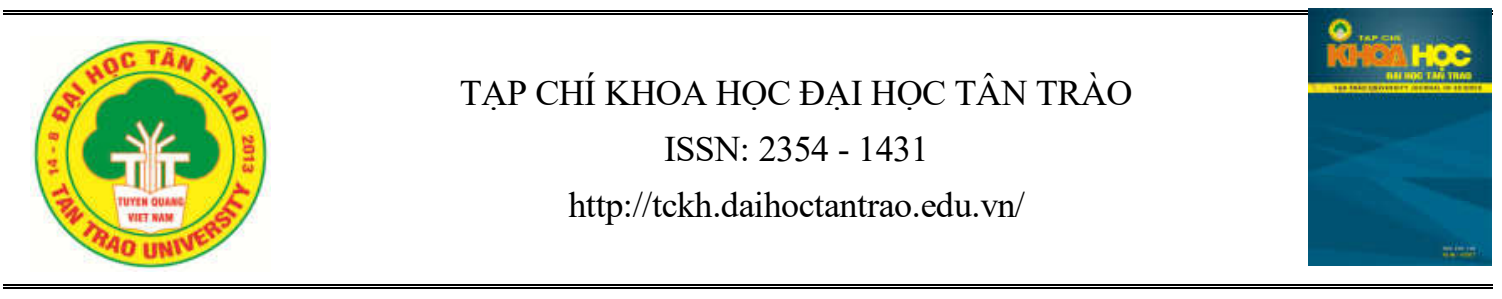

\title{
Giải pháp nâng cao hiệu quả rèn kỹ năng mềm cho sinh viên các trường đại học
}

\author{
Phạm Thị Quyên ${ }^{a^{*}}$ \\ ${ }^{a}$ Truò̀ng Đại học Tân Trào \\ *Email: phamquyen1985@gmail.com
}

\section{Thông tin bài viết}

Ngày nhận bài:

19/12/2019

Ngày duyệt đăng:

$10 / 3 / 2020$

Tù khóa:

Kỹ năng mềm; phát triển kỹ năng mềm cho sinh viên; biện pháp rèn kỹ năng mềm.

\section{Tóm tắt}

Rèn kỹ năng mềm cho sinh viên là một yêu cầu khách quan đang được quan tâm nhằm nâng cao chất lượng đào tạo, đáp ứng nhu cầu thị trường lao động. Bài viết đề cập tới một số khái niệm có liên quan tới kỹ năng mềm, vai trò và biện pháp nâng cao hiệu quả rèn kỹ năng mềm cho sinh viên các trường đại học.

\section{1. Đặt vấn đề}

Hoạt động nghề nghiệp phụ thuộc vào năng lực và trình độ được đào tạo của mỗi cá nhân, năng lực của mỗi cá nhân được cấu trúc bởi hai thành phần, đó là năng lực cốt lõi và năng lực chung. Phần năng lực cốt lõi là hệ thống kiến thức chuyên môn và kỹ năng $(\mathrm{KN})$ nghề nghiệp; phần năng lực chung là những kiến thức, $\mathrm{KN}$ bổ trợ giúp cho kiến thức, $\mathrm{KN}$ chuyên môn được tiến hành có hiệu quả. Thực tế cho thấy, sự thành đạt của mỗi người phụ thuộc rất nhiều vào hệ thống $\mathrm{KN}$ bổ trợ hay còn gọi là kỹ năng mềm (KNM), theo nhà nghiên cứu Rani $\mathrm{S}$. cho rằng, sự thành đạt của con người do $\mathrm{KNM}$ và chỉ số $\mathrm{EQ}$ quyết định tới $75 \%$ [7, tr. 55]. Nhờ có KNM mà tư duy của mỗi cá nhân trở nên linh hoạt hơn, sáng tạo hơn, mềm dẻo hơn; đồng thời có cơ hội hợp tác, chia sẻ cùng người khác, thích ứng với thế giới việc làm luôn biến đổi. KNM không tồn tại độc lập mà nó gắn kết với $\mathrm{KN}$ chuyên môn tạo nên năng lực hành động của mỗi người hay không do tư chất của cá nhân quyết định mà được hình thành, phát triển và ngày càng hoàn thiện thông qua quá trình trải nghiệm nghề nghiệp, học tập, rèn luyện và thực tế cuộc sống.
Trong những năm qua, đi đôi với sự phát triển về quy mô và số lượng đào tạo ở các trường đại học dẫn tới sự tăng trưởng nóng về nguồn nhân lực có trình độ cao. Tuy nhiên, vấn đề chất lượng đào tạo mới chỉ tập trung vào cung cấp kiến thức, chưa chú ý nhiều đến rèn các $\mathrm{KN}$ chuyên môn, đặc biệt là $\mathrm{KNM}$ dẫn tới tình trạng sinh viên (SV) hạn chế về $\mathrm{KN}$ giao tiếp, $\mathrm{KN}$ làm việc nhóm, $\mathrm{KN}$ thuyết phục... $\mathrm{KN}$ thích ứng, tự ứng phó với những thay đổi của nghề nghiệp và thị trường lao động. Bởi vậy, giảng viên $(\mathrm{GV})$ cần thực hiện các biện pháp nâng cao chất lượng rèn $\mathrm{KNM}$ cho $\mathrm{SV}$, từ đó nâng cao chất lượng đào tạo của nhà trường.

\section{Nội dung nghiên cứu}

\subsection{Một số khái niệm có liên quan}

Để làm cơ sở nghiên cứu nội dung nâng cao chất lượng rèn $\mathrm{KNM}$ cho $\mathrm{SV}$ các trường đại học, chúng tôi sử dụng khái niệm công cụ sau:

2.1.1. Kỹ năng: Có nhiều khái niệm về $\mathrm{KN}$ nghiêng về mặt kỹ thuật của hành động hay khả năng thể hiện ở kết quả của hành động. Song hầu hết các tác giả đều cho rằng, $\mathrm{KN}$ là sản phẩm của quá trình tư duy, tích lũy kinh nghiệm của con người trong hoạt 
động thực tiễn. KN là bậc cao của thao tác kỹ thuật mang tính thành thạo, chủ động và có kết quả ổn định. Nói cách khác, KN là năng lực của con người sử dụng tri thức, các phương tiện, cách thức hành động một cách linh hoạt, sáng tạo, phù hợp với hoàn cảnh, điều kiện biến động của thực tiễn, hành động luôn gắn với mục tiêu và điều kiện xác định, chính mục tiêu, điều kiện xác định quyết định loại thao tác và trật tự thao tác trong hành động. Khi điều kiện biến động với các mức độ khác nhau thì thao tác và trật tự của chúng cũng phải thay đổi tương ứng. $\mathrm{KN}$ bao giờ cũng gắn với một hoạt động cụ thể ở các lĩnh vực khác nhau. Kế thừa những quan niệm trên, chúng tôi đưa ra khái niệm: Kỹ năng là một dạng hành động của con người vận dụng sáng tạo tri thức, kinh nghiệm và cách thức hành động vào hoạt động thực tiễn trong các điều kiện và hoàn cảnh cụ thể, thực hiện có kết quả theo mục đích hay tiêu chí đã đặt ra [2, tr. 12].

2.1.2. Kỹ năng sống (KNS): Có nhiều quan niệm khác nhau, trên cơ sở tiếp thu, kế thừa những quan niệm khác nhau về KNS, chúng tôi xác định: KNS là hệ thống các kỹ năng cơ bản giúp cá nhân tồn tại và thích ứng trong cuộc sống; những kỹ năng này giúp cá nhân thể hiện được chính mình cũng như tạo ra những nội lực cần thiết để thích nghi và phát triển trong các điều kiện khác nhau của cuộc sống [2, tr. 12-13].

2.1.3. Kỹ năng cứng: $\mathrm{KN}$ này thường được hiểu là những kiến thức, đúc kết và thực hành có tính chất kỹ thuật nghề nghiệp và liên quan đến chỉ số thông minh (IQ) của cá nhân. $\mathrm{KN}$ cứng là $\mathrm{KN}$ nền tảng, được đo, đào tạo từ nhà trường. $\mathrm{KN}$ cứng được phát triển dần từ mức độ thấp lên các mức độ cao hơn, thông qua quá trình dạy học, tự học, thực hành, hoạt động thực tiễn một cách hệ thống. $\mathrm{KN}$ cứng thường xuất hiện trên bản lý lịch về khả năng học vấn, kinh nghiệm và sự thành thạo về chuyên môn của mỗi con người. Như vậy, $\mathrm{KN}$ cứng là những $\mathrm{KN}$ nghề nghiệp thể hiện trình độ học vấn hay bằng cấp và chứng chỉ, kinh nghiệm và sự thành thạo về chuyên môn qua hành vi, hành động của mỗi con người $[1, \operatorname{tr} 8]$.

2.1.4. Kỹ năng mềm: Là thuật ngữ dùng để chỉ các KN quan trọng trong cuộc sống, thể hiện cách sống, cách tích lũy kinh nghiệm từ cuộc sống và được xem là chìa khóa vàng dẫn đến thành công của mỗi con người. KNM có được từ hoạt động thực tế cuộc sống, hoạt động nghề nghiệp và liên quan đến chỉ số về trí tuệ cảm xúc (EQ) của con người. KNM là khả năng làm cho hành vi và sự thay đổi của mình phù hợp với cách ứng xử tích cực của con người, kiểm soát, quản lý có hiệu quả các nhu cầu và những thách thức trong cuộc sống.

Theo Radhika Ch. KNM được định nghĩa: "Là kỹ năng giao tiếp giữa người này với người khác và $\mathrm{KN}$ cư xử mà con người cần để có thể ứng dụng được những kiến thức cũng như các $\mathrm{KN}$ chuyên môn ở nơi làm việc" [6, tr.25]. Theo Rani $\mathrm{S}$ : [7, tr. 55], "KNM là những kỹ năng mà con người sử dụng để hành xử, làm việc với nhau, giải quyết các mâu thuẫn, thân thiện, lạc quan và thuyết phục người khác". Tác giả Alex K. lại cho rằng: "KNM là các $\mathrm{KN}$ giúp con người sử dụng các $\mathrm{KN}$ chuyên môn và kiến thức thực tế hiệu quả hơn, giúp bạn tiến xa hơn trong một nghề nghiệp nào đó" $[4$, tr. 36$]$. Khái niệm KNM có nhiều cách tiếp cận khác nhau, chúng tôi sử dụng khái niệm: KNM là hệ thống các $\mathrm{KN}$ cơ bản được thực hiện tự giác dựa trên tri thức về công việc, khả năng hòa nhập xã hội, thái độ và hành vi ứng xử hay tương tác với xã hội, cộng đồng, bạn bè, đồng nghiệp, đối tác, tổ chức của mỗi cá nhân nhằm phát huy tối đa hiệu quả công việc và thành đạt trong cuộc sống.

\subsection{Vai trò của KNM đối vói SV các trưòng đại học}

KNS theo nghĩa rộng là giúp con người có khả năng tồn tại và thích ứng trước cuộc sống thực tế, là một năng lực quan trọng để con người làm chủ được bản thân và chung sống, thích ứng với mọi người xung quanh, với xã hội; với môi trường, với thiên nhiên,... một cách hiệu quả.

KNM giúp cá nhân tồn tại, thành công trong công việc hay trong những mối quan hệ với người khác, hướng đến giúp mỗi cá nhân thích nghi trong công việc, tương tác hiệu quả với người khác và thúc đẩy công việc đạt hiệu quả cao. KNM phụ thuộc vào nghề nghiệp, đặc biệt là đối tượng của nghề nghiệp hướng đến. Mỗi con người để có một công việc, tất yếu họ phải được đào tạo đủ $\mathrm{KN}$ chuyên môn ( $\mathrm{KN}$ cứng) đáp ứng các yêu cầu tối thiểu của vị trí công việc. $\mathrm{KN}$ cứng giúp con người bước vào ngưỡng cửa của nghề nghiệp, vị trí làm việc,... Song các KNM mới là thứ giúp cho họ vững vàng trong nghề nghiệp, giữ được việc làm và thành đạt trong cuộc sống. Thái độ đối với công việc, giao tiếp, trí tuệ tình cảm và các đức tính, giá trị cá nhân khác là những KNM không thể thiếu để mỗi người phát triển nghề nghiệp. KNM luôn đồng hành cùng $\mathrm{KN}$ cứng và $\mathrm{KNM}$ bổ trợ cho các $\mathrm{KN}$ cứng được phát huy, phát triển. Nhờ có sự kết hợp giữa $\mathrm{KN}$ cứng và KNM giúp cho mỗi cá nhân có được sự thành công trong quá trình phát triển nghề nghiệp của mình, 
họ khẳng định được vị trí của mình trong tổ chức, xã hội [3, tr. 27-29].

Xã hội tri thức đòi hỏi con người ngoài năng lực chuyên môn cần phải có hệ thống các KNM để thích ứng với môi trường luôn biến đổi của thị trường lao động và thế giới việc làm. Trong xã hội tri thức, KNM giúp con người có khả năng giải quyết linh hoạt, sáng tạo các vấn đề của cuộc sống và nghề nghiệp đặt ra, sử dụng hiệu quả kiến thức, $\mathrm{KN}$ cứng trong lao động nghề nghiệp, phát triển chuyên môn và dịch chuyển nghề nghiệp trong thế giới việc làm luôn thay đổi.

Trong nghiên cứu về: "Vai trò của giáo dục đối với sự hình thành các KNM" tác giả Greenberg A.D. và Nilssen A.H. đã đưa ra kết luận: Các nhà trường phải trang bị tốt hơn cho người học những kiến thức và KNM cần thiết để họ có thể sẵn sàng làm việc sau khi ra trường [5, tr.28]. Vì vậy, các chương trình phát triển $\mathrm{KMN}$ trong giáo dục nghề nghiệp cần tích hợp vào các chương trình dạy $\mathrm{KN}$ nghề nghiệp nhằm thực hiện mục tiêu kép, đó là tăng cường cho người học cơ hội học tập, chuẩn bị bước vào thế giới công việc bằng việc tạo cho họ đầu vào là những $\mathrm{KN}$ nghề nghiệp được đào tạo. Đồng thời, tăng tính hiệu quả và sự phù hợp của người học với các $\mathrm{KN}$ nghề được đào tạo (đáp ứng thị trường lao động, mong muốn của cá nhân về sự thăng tiến, thu nhập...). Như vậy, rèn KNM cho SV là đáp ứng yêu cầu khách quan của xã hội.

\subsection{Một số biện pháp nâng cao hiệu quả rèn KNM cho $S V$}

Để nâng cao hiệu quả rèn $\mathrm{KNM}$ cho $\mathrm{SV}, \mathrm{GV}$ cần sử dụng phối kết hợp nhiều biện pháp tùy thuộc vào nội dung, điều kiện, hoàn cảnh của nhà trường và $\mathrm{SV}$. Đây là cách thức, con đường tổ chức các hoạt động rèn $\mathrm{KNM}$ cho $\mathrm{SV}$, giúp $\mathrm{KNM}$ của $\mathrm{SV}$ tiến triển theo chiều hướng tăng lên từ mức độ thấp đến mức độ cao hơn, từ chưa hoàn thiện đến hoàn thiện hơn $[3$, tr. 27 29]. Trong nghiên cứu này chúng tôi đề xuất 3 biện pháp sau.

2.3.1. Biên soạn tài liệu huớng dẫn giảng dạy KNM

Biên soạn tài liệu hướng dẫn giảng dạy $\mathrm{KNM}$ nhằm trang bị cho $\mathrm{GV}$ những tri thức, đặc điểm các KNM; phát triển chương trình, thiết kế nội dung bài giảng, sử dụng các phương pháp giảng dạy, đánh giá phù hợp. Ngoài ra, thông qua tài liệu bồi dưỡng giúp GV tự bồi dưỡng năng lực giảng dạy $\mathrm{KNM}$ và coi như là cẩm nang để thực hiện tốt nhiệm vụ giảng dạy KNM cho SV.
Mục tiêu của tài liệu được biên soạn là trợ giúp cho GV thực hiện nhiệm vụ giảng dạy, tích hợp giảng dạy KNM cho SV. Thực hiện biện pháp này gồm 2 nội dung sau:

1/ Tài liệu hướng dẫn thiết kế module dạy học KNM gồm nội dung: - Hướng dẫn cách xây dựng các module dạy học KNM; - Hướng dẫn xây dựng các tiểu module, tài liệu cần giúp $\mathrm{GV}$ hiểu về module, tiểu module dạy học $\mathrm{KNM}$ và cách lắp ráp các tiểu module với nhau để đạt được mục tiêu dạy học; - Hướng dẫn cách xác định hệ vào của module: Hệ vào của module phải được thiết kế dựa trên tính vấn đề của nội dung dạy học và năng lực nhận thức, trình độ đạt được ở $\mathrm{SV}$, hệ vào của modul được thiết kế chuẩn có tác dụng định hướng năng lực và định hướng hành động cho SV trong quá trình thực hiện thân module và hệ ra của module; - Hướng dẫn xây dựng thân module: Thân module chứa đựng các hành động học tập, rèn luyện của $\mathrm{SV}$ dưới vai trò định hướng của $\mathrm{GV}$, vì vậy đòi hỏi việc thiết kế thân module phải khoa học, logic và đảm bảo tính sư phạm, tính mục đích khi rèn KNM cho SV; - Hướng dẫn xây dựng hệ ra của module, bởi hệ ra của module có tác dụng đánh giá sơ bộ kiến thức, $\mathrm{KN}$ của $\mathrm{SV}$ đã đạt được thông qua quá trình tham gia hoạt động ở thân module; - Hướng dẫn xây dựng hệ thống thông tin phản hồi, hệ thống bài tập thực hành rèn luyện $\mathrm{KNM}$ cho $\mathrm{SV}$ và các biện pháp, kỹ thuật dạy học theo module, những điểm GV cần lưu ý trong quá trình tổ chức thực hiện.

2/ Hướng dẫn giảng dạy các module KNM, bao gồm: - Hướng dẫn xác định các module giảng dạy $\mathrm{KNM}$, nội dung phát triển $\mathrm{KNM}$ cho $\mathrm{SV}$ phù hợp để đạt tới các mục tiêu đã đề ra; - Hướng dẫn GV lựa chọn các hình thức tổ chức dạy học, phương pháp giảng dạy và học tập phù hợp giúp chuyển tải được nội dung môn học, nội dung phát triển KNM và đạt tới mục tiêu; - Hướng dẫn GV xác định các phương pháp đánh giá phù hợp (động viên, khuyến khích, đánh giá đúng trình độ của người học). Đề ra tiêu chuẩn, tiêu chí đánh giá cụ thể, công khai gồm: + Đánh giá thường xuyên: Kiểm tra thường ngày trên lớp thông qua phát biểu, thảo luận, kết quả bài tập cá nhân, nhóm được giao, kết quả thực hành,...; + Đánh giá định kỳ: Được thực hiện bằng cách giao bài tập cho cá nhân (hoặc nhóm) /tuần (tháng). Bài tập lớn (tiểu luận) học kỳ (hình thức bài tập nghiên cứu hoặc nhóm dự án); + Đánh giá cuối kỳ (hoặc hết môn): Được thực hiện bằng bài tổng luận môn học (mức độ biết phát hiện vấn đề, biết vận dụng, giải quyết vấn đề). Tất các 
những hướng dẫn nêu trên phải được GV thể hiện trong đề cương môn học và chương trình đào tạo của từng chuyên ngành.

Điều kiện thực hiện biện pháp là chuyên gia biên soạn tài liệu hướng dẫn về giảng dạy KNM phải là người am hiểu sâu về khoa học kinh tế, tài chính, quản trị kinh doanh, hiểu biết sâu về $\mathrm{KNM}$ và quá trình giảng dạy, rèn luyện, phát triển KNM cho SV. Tài liệu phải được biên soạn theo từng phần, dễ hiểu và thuận lợi cho GV tự nghiên cứu, vận dụng hiệu quả. Do đó, GV cần nhận thức rõ ràng, đầy đủ về tầm quan trọng và vai trò của năng lực giảng dạy nói chung, năng lực giảng dạy KNM nói riêng đối với việc nâng cao chất lượng đào tạo của nhà trường. Từ đó có ý thức tự nghiên cứu, tự bồi dưỡng, rèn luyện năng lực giảng dạy trong suốt quá trình dạy học của mình.

\subsubsection{Tổ chức dạy học tích hợp phát triển KNM} cho $S V$

KNM chỉ được hình thành, phát triển thông qua hoạt động, bằng hoạt động. Vì vậy, trong mỗi giờ lên lớp bất kỳ một môn học nào, GV đều phải quan tâm rèn luyện cho $\mathrm{SV}$ hệ thống các $\mathrm{KNM}$ cần thiết, việc làm đó phải được tiến hành ngay từ khâu thiết kế bài giảng. Biện pháp này giúp $\mathrm{GV}$ thông qua nội dung môn học chuyên môn, tích hợp KNM trong quá trình thiết kế bài giảng phần phương pháp, biện pháp, kỹ thuật dạy học, tạo môi trường, tăng cường rèn luyện KNM cho SV. Môi trường học tập các môn học được thiết kế theo hướng tăng cường hoạt động, giúp SV có cơ hội được thực hành các KNM, qua đó phát triển KNM cho SV. Để giờ học thành công, đạt hiệu quả thì ngay từ khâu thiết kế bài giảng $\mathrm{GV}$ đã phải thể hiện tường minh từng nội dung hoạt động, từng $\mathrm{KN}$ được rèn luyện, tập luyện trong các giờ học sẽ tiến hành. Việc thiết kế bài giảng có tích hợp KNM cho SV là cơ sở để GV tổ chức bài học thành công, hiệu quả, giúp GV biến những mục tiêu về phát triển KNM cho $\mathrm{SV}$ thành hành động thực tiễn ở SV.

KNM không tồn tại độc lập mà nó có mối quan hệ mật thiết với $\mathrm{KN}$ chuyên môn. Vì vậy, $\mathrm{GV}$ cần có $\mathrm{KN}$ tổ chức dạy học theo hướng tích hợp KNM để phát triển $\mathrm{KNM}$, việc phát triển $\mathrm{KNM}$ cần được phối kết hợp trong quá trình hình thành và phát triển $\mathrm{KN}$ chuyên môn cho SV. Dạy học theo hướng tích hợp phát triển $\mathrm{KNM}$ cho $\mathrm{SV}$ nhằm tận dụng kép thời gian và nguồn lực trong các môn học. Mặt khác, tích hợp việc học kiến thức chuyên ngành và phát triển $\mathrm{KNM}$ giúp SV phát triển kiến thức chuyên ngành sâu hơn. Về phía GV sẽ đưa các KNM phù hợp vào chuẩn đầu ra của môn học do GV phụ trách, tạo cơ hội cho SV phát triển các $\mathrm{KN}$ này thông qua hoạt động giảng dạy và học tập trên lớp.

Lồng ghép, tích hợp phát triển $\mathrm{KNM}$ cho $\mathrm{SV}$ trong dạy học môn học theo các bước sau:

- Bước 1: Công bố mục tiêu bài học và mục tiêu phát triển KNM cần lồng ghép, tích hợp để $\mathrm{SV}$ định hướng hành động. GV cần nêu rõ mục tiêu kiến thức $\mathrm{KN}$ chuyên môn và các $\mathrm{KNM}$ mà $\mathrm{SV}$ cần rèn luyện qua bài học. Định hướng cách thức rèn luyện cho $\mathrm{SV}$ qua quá trình tham gia hoạt động và lĩnh hội, rèn luyện kiến thức, $\mathrm{KN}$ chuyên môn, trải nghiệm thực tế nghề nghiệp và cuộc sống.

- Buớc 2: Tạo môi trường hoạt động học tập để SV lĩnh hội kiến thức, $\mathrm{KN}$ chuyên môn và rèn luyện các KNM GV có thể sử dụng những biện pháp kĩ thuật hiện đại để tạo môi trường học tập, rèn luyện cho SV (ví dụ như phương pháp xử lý tình huống, nghệ thuật phá vỡ tảng băng, phương pháp tổ chức trò chơi, kỹ thuật phòng tranh hay sử dụng các kỹ thuật khác...) với mục đích tạo ra tâm lý thoải mái, tự tin, khích lệ SV tích cực tham gia hoạt động, rèn luyện để phát triển kiến thức, $\mathrm{KN}$ chuyên môn và $\mathrm{KNM}$.

- Buớc 3: Tổ chức các tình huống dạy học để hình thành tri thức, $\mathrm{KN}$ chuyên môn và $\mathrm{KNM}$ cho $\mathrm{SV}$. Bài học được thực hiện theo các tình huống dạy học, trong đó chứa đựng tình huống có vấn đề buộc người học phải tích cực tham gia để rèn luyện $\mathrm{KNM}$, thông qua các tình huống dạy học $\mathrm{GV}$ có nhiệm vụ thu hút người học hoạt động trải nghiệm để lĩnh hội kiến thức, $\mathrm{KN}$ chuyên môn và KNM.

Khi tổ chức bài dạy theo quan điểm tích hợp, $\mathrm{GV}$ không chỉ chú trọng nội dung kiến thức tích hợp mà còn phải quan tâm tổ chức một hệ thống hoạt động, thao tác tương ứng giúp tổ chức, dẫn dắt người học từng bước thực hiện để hình thành năng lực và KNM. Bài dạy lồng ghép, tích hợp phát triển KNM phải là một giờ học có những hoạt động phức hợp đòi hỏi sự tích hợp các kiến thức, $\mathrm{KN}$ chuyên môn, $\mathrm{KNM}$ để giải quyết tình huống nghề nghiệp và cuộc sống.

Điều kiện thực hiện biện pháp là $G V$ phải giỏi về kiến thức chuyên môn giảng dạy, am hiểu sâu về $\mathrm{KNM}$; có $\mathrm{KN}$ sư phạm tốt, am hiểu và làm chủ các phương pháp, phương tiện, biện pháp kỹ thuật dạy học (đặc biệt là sử dụng phương tiện dạy học hiện đại) chiếm ưu thế trong việc phát triển $\mathrm{KNM}$ cho $\mathrm{SV}$. Ngoài ra, GV phải là người có $\mathrm{KN}$ thiết kế kịch bản rèn luyện $\mathrm{KNM}$ cho $\mathrm{SV}$ trong quá trình thiết kế bài học. Để tổ chức dạy học lồng ghép, tích hợp phát triển 
KNM thành công đòi hỏi $\mathrm{GV}$ phải biên soạn được giáo án tích hợp nội dung phát triển KNM phù hợp với trình độ của người học, điều kiện thực tiễn của cơ sở đào tạo, nhưng vẫn đảm bảo mục tiêu, nội dung và thời gian đã quy định.

\subsection{3. Đổi mới hoạt động đánh giá kết quả học tập} của SV theo tiếp cận năng lục

Điều này tạo động lực cho quá trình rèn luyện $\mathrm{KN}$ chuyên môn và $\mathrm{KNM}$ của của $\mathrm{SV}$ đạt hiệu quả cao, kích thích SV tích cực vươn lên trong học tập, rèn luyện để phát triển toàn diện về năng lực, phẩm chất nhân cách. Thông qua hoạt động đánh giá giúp $G V$ và cá nhân SV quan tâm nhiều hơn đến việc SV "làm được gì?", "vận dụng được gì?" từ những điều đã học trong thực tiễn học tập, lao động nghề nghiệp và cuộc sống. Đổi mới hoạt động kiểm tra, đánh giá sẽ tạo động lực cho quá trình dạy học, giáo dục phát triển và $\mathrm{SV}$ tích cực rèn luyện $\mathrm{KN}$ chuyên môn, $\mathrm{KNM}$, giúp SV khẳng định năng lực của mình, từ đó có kế hoạch tự hoàn thiện bản thân.

Đổi mới cách đánh giá phải bắt đầu từ đổi mới cách dạy, $\mathrm{GV}$ cần đổi mới từ thiết kế đề cương chi tiết môn học, đổi mới hình thức tổ chức dạy học, phương pháp dạy học theo tiếp cận năng lực, quan tâm đến việc giúp $\mathrm{SV}$ hiểu và vận dụng kiến thức nhiều hơn là yêu cầu $\mathrm{SV}$ phải học thuộc lòng và ghi nhớ một cách máy móc những kiến thức đã học.

Trước tiên $\mathrm{GV}$ cần thiết kế được hệ thống các mục tiêu học tập có tích hợp KNM theo 3 cấp độ khác nhau: - Nhận biết, tái hiện; - Hiểu, vận dụng; vận dụng thành thạo trong những tình huống khác nhau; - Giải quyết vấn đề dựa trên sự phân tích, tổng hợp, đánh giá thể hiện quan điểm cá nhân.

Từ hệ thống mục tiêu ở 3 cấp độ nêu trên, GV thiết lập ma trận ngân hàng câu hỏi theo 3 cấp độ khác nhau để đánh giá năng lực của $\mathrm{SV}$ qua hình thức đánh giá. Hình thức này càng được đa dạng hóa bao nhiêu thì kết quả đánh giá càng khách quan bấy nhiêu. Vì vậy, GV cần đa dạng hóa các hình thức đánh giá như: Đánh giá cá nhân, đánh giá kết quả của nhóm, đánh giá tự học, chuẩn bị bài ở nhà, đánh giá tiểu luận, bài tập, kết quả thực hành, kiểm tra định kỳ, thi tổng kết cuối kỳ... Mục đích đánh giá không chỉ là điểm số mà là sự tiến bộ về năng lực của $\mathrm{SV}$. Quá trình dạy học phải bám sát năng lực của $S V$, tùy trình độ và đặc điểm hiện có của $\mathrm{SV}$ mà cách thức tác động sẽ khác nhau để SV thích học, hứng thú với hoạt động học tập và tích cực học tập. Hay nói cách khác, để đảm bảo đánh giá theo năng lực $\mathrm{GV}$ cần tiến hành tổ chức dạy học phân hóa một cách hiệu quả. Cho nên, những thông tin phản hồi thường xuyên của $\mathrm{GV}$ đối với $\mathrm{SV}$ cần được thực hiện một cách nghiêm túc giúp $\mathrm{SV}$ điều chỉnh kịp thời, qua đó $G V$ cũng điều chỉnh về hình thức tổ chức, phương pháp dạy học phù hợp.

Điều kiện thực hiện biện pháp này là những quy định về đánh giá kết quả học tập của SV theo tiếp cận năng lực cần được thể hiện ngay khi thiết kế chương trình dạy học, $\mathrm{GV}$ và cán bộ quản lý cần thay đổi nhận thức về phương pháp dạy học và kiểm tra, đánh giá hiện nay. GV cần nắm vững quy trình, cách thức tiến hành kiểm tra, đánh giá theo tiếp cận năng lực. Nhà trường cần có hệ thống văn bản hướng dẫn và quản lý hoạt động kiểm tra, đánh giá kết quả học tập, rèn luyện của $S V$ theo tiếp cận năng lực.

\section{Kết luận}

Trên cơ sở nghiên cứu một số vấn đề lý luận về KNM, chúng tôi đã đề xuất 3 biện pháp với mục đích nâng cao hiệu quả việc rèn $\mathrm{KNM}$ cho $\mathrm{SV}$ các trường đại học. Để các biện pháp được thực hiện mang lại kết quả cần có sự đồng thuận, nhất quán về nhận thức trong cán bộ, $\mathrm{GV}$, viên chức, $\mathrm{SV}$ nhà trường về tầm quan trọng rèn $\mathrm{KNM}$ cho $\mathrm{SV}$. Cần xác định nội dung, hình thức và phương pháp đào tạo, giáo dục phù hợp, hiệu quả để phát triển kỹ năng nói chung, KNM nói riêng cho $\mathrm{SV}$, góp phần nâng cao chất lượng đào tạo của nhà trường. Bên cạnh đó cần tạo điều kiện đầu tư kinh phí, cơ sở vật chất, trang thiết bị để nhà trường tổ chức các hoạt động giáo dục, dạy học theo hướng chủ động, sáng tạo và tăng cường hoạt động của $\mathrm{SV}$, tạo môi trường để $\mathrm{SV}$ phát triển kỹ năng ( $\mathrm{KN}$ nghề nghiệp, KNM), nâng cao chất lượng đào tạo, thực hiện đào tạo theo yêu cầu thị trường lao động. Ban hành cơ chế chính sách giúp gắn kết chặt chẽ giữa nhà trường với doanh nghiệp; các cơ quan, tổ chức trong quá trình đào tạo; thực hiện đào tạo gắn với yêu cầu xã hội trong thời kì mới.

\section{TÀI LIÊU THAM KHẢO}

1. Nguyễn Thị Mỹ Lộc, Đinh Thị Kim Thoa (2012), Hoạt động giáo dục giá trị sống và kỹ năng sống cho hoc sinh trung học, Tài liệu tập huấn/bồi dưỡng giáo viên của Bộ GD\&ĐT tập 1, trang 8, Hà Nội.

2. Huỳnh Văn Sơn (2009), Nhập môn kĩ năng sống, NXB Giáo dục Việt Nam, Hà Nội, trang 12-13.

3. Tạ Quang Thảo (2014), Phát triển kỹ năng mềm cho sinh viên các trường đại học, cao đẳng đáp úng yêu cầu của thị truờng lao động trong giai đoạn hiện 
nay, Tạp chí giáo dục, số 329 kì 01, trang 27, 28, 29, tháng 3/2014.

4. Alex K. (2010), Soft skills: Knowing yourself \& Know the World, New Delhi: S. Chnd. \& Company Ltd, page 36, 2010.

5. Greenberg A. D. \& Nilssen A.H. (2015), The role of education in building soft skills, Wain house Research, LLC, page 28, 2015.
6. Radhika Ch (2013), Soft skills - A buzz word in the new millennium, Indian Streams Research Journal, Volume 3, Issue 7, ISSN 2230-7850, page 25, 2013.

7. Rani S (2010), Need and importance of soft skills in students, Sri Sarada College for Women, Salem - 636016, page 55, 2010.

\section{Solutions to improve efficiency of soft skills for students in universities}

Pham Thi Quyen

\section{Article info}

Recieved:

19/12/2019

Accepted:

$10 / 3 / 2020$

Keywords:

Soft skills; improve soft skills for students;

solution to improve soft skills.

\begin{abstract}
Training soft skills for students is an objective mission that is caring to enhance the training quality and meet the social demand. This article mentions some definitions that are related to soft skills, function and solution to improve efficiency of soft skills for students in universities.
\end{abstract}

